\title{
Satisfaction with care as a quality-of-life predictor for stroke patients and their caregivers
}

\author{
Jane M. Cramm • Mathilde M. H. Strating • \\ Anna P. Nieboer
}

Accepted: 26 December 2011/Published online: 10 January 2012

(C) The Author(s) 2012. This article is published with open access at Springerlink.com

\begin{abstract}
Purpose We sought to identify indicators associated with the quality of life (QoL) of stroke patients and caregivers. Methods The cross-sectional study was conducted at nine Dutch stroke service facilities involving 251 stroke patients and their caregivers. We used the EuroQol (EQ-5D) and Satisfaction with Stroke Care questionnaires, and included the variables (1) disability at hospital admission, (2) length of hospital stay, (3) demographic data, and (4) caregivers' relationship with stroke patients. The Actor-Partner (patient-caregiver) Interdependence Model (APIM) was used to examine dependence between patients' and caregivers' QoL scores through dyad membership.

Results Patients' age was significantly related to their QoL, and caregivers' age and educational level were significantly related to their QoL. Patients' disability on hospital admission and length of stay were associated with patients' QoL, and their disability on admission was related to caregivers' QoL. No relationship was found between length of stay and caregivers' QoL. Satisfaction with care was associated with both patients' and caregivers' QoL.

Conclusions The APIM distinguished the different roles of patients and caregivers while acknowledging the interdependence of their QoL scores. Satisfaction with care was
\end{abstract}

\footnotetext{
J. M. Cramm ( $\varangle)$ · M. M. H. Strating · A. P. Nieboer Institute of Health Policy and Management (iBMG), Erasmus University Rotterdam, Burgemeester Oudlaan 50, 3000 DR Rotterdam, The Netherlands

e-mail: cramm@bmg.eur.nl

M. M. H. Strating

e-mail: strating@bmg.eur.nl

A. P. Nieboer

e-mail: nieboer@bmg.eur.nl
}

identified as important indicator of stroke patients' and caregivers' QoL.

Keywords Stroke - Quality of life - Caregiver . Satisfaction with care $\cdot$ The Actor-Partner Interdependence Model

\section{Introduction}

Acute strokes are highly prevalent in Western countries. A stroke diagnosis is associated with high mortality and leads to increased morbidity, including chronic physical impairments and functional limitations that affect quality of life (QoL) [1-5]. Many patients, families, and professionals consider long-term QoL central for recovery from stroke [6]. Stroke affects both patients and caregivers. Informal caregivers are the backbone of the services provided to stroke patients [7, 8], and the task is known to decrease their QoL [914]. Care for stroke patients can be stressful and frequently represents a considerable long-term burden [15-17]. Caregivers must attend to patients' mobility, personal care, communication, cognitive impairment, depression, and personality changes [13]. People involved in this kind of dyadic relationship strongly influence each other's cognitions, emotions, and behaviors; patients' and caregivers' QoL are thus correlated and interdependent [18]. Consequently, the attributes and behaviors of one dyad member can affect the outcomes of the other [19]. The simultaneous investigation of stroke patients' and their caregivers' QoL is thus the most appropriate means of increasing our understanding of the relationship and the mechanisms through which stroke is related to the QoL of both parties [18, 19].

As an important predictor of quality of care, patient satisfaction has increasingly received attention in the 
measurement of stroke outcome [20, 21]. Higher satisfaction with stroke care could alleviate both patient stress and caregiver stress, thereby positively affecting their QoL. The delivery of hospital stroke care that supports patients and caregivers by meeting their needs and demands is expected to positively affect their QoL [15, 20]. Earlier research showed that QoL of patients depends on age, length of hospital stay, and severity of disability in the acute phase [2-4]. We investigated the relationship between these indicators and patients' QoL. There is interdependence in a relationship when observations of two individuals - in our case stroke patients and their caregivers-are linked or correlated [19]. We expect linkages and interdependence of observation among stroke patients and their caregivers, which calls for unit of analysis of the dyad rather than the individual. Therefore, our analysis additionally focused on the exploration of the dyad effect, whereby caregivers' QoL is also expected to depend on the above patient variables $[7,11,13]$. The aim of this study is to identify the relationship between length of hospital stay and severity of disability of stroke patients with QoL of both stroke patients and their caregivers. Furthermore, this study specifically aims to identify the relationship between patients' and caregivers' satisfaction with stroke care and their QoL.

\section{Methods}

Our cross-sectional study was conducted at nine stroke service facilities in the Netherlands [22, 23]. These stroke service facilities aim to admit all patients suspected of having a stroke to the (university) hospital for diagnosis, followed by fast transition to, preferably, home, and otherwise to a rehabilitation centre or a specialized nursing home. These stroke services had a well-developed multidisciplinary approach to patient care. Professional interventions included disseminated guidelines and working according to protocols. Patient interventions to improve adjustment and recovery included information booklets/ records, education, training and counseling, and social or emotional support. The basic assumption behind this stroke service is that this integrated care model will result in a more effective as well as more efficient health care for stroke patients. These stroke services were all collecting data of patients and caregivers to evaluate and compare their stroke care. Because the measurements of satisfaction with care were part of daily practice and were initiated and implemented by stroke service facilities rather than the research team, the Medical Ethics Committee determined that the study did not need ethical approval. The managers of each facility granted access to subjects. All persons gave their informed consent prior to their inclusion in the study.
Subjects

Stroke patients were identified through the stroke service facilities. All the patients participating in this study were inpatients only. Patients who were subsequently diagnosed with transient ischemic attack (TIA) instead of stroke were excluded, which led to 915 stroke patients. Ninety-one of these patients died during their hospital stay, which makes the sample 824 patients. Caregivers were identified through the participating stroke patients. Caregivers are those who provide structured care voluntarily and for free to the stroke patient. It involves providing more care than usual in a personal relationship and consists of tasks that healthy people could normally do themselves. We received a total of 377 questionnaires from patients (out of 824; response rate $46 \%$ ) and 332 from caregivers (out of 824; response rate $40 \%$; assuming that all patients had a caregiver). Since we are interested in couples of patients and caregiver only, we excluded questionnaires of patients and caregiver that cannot be connected. This led to a final sample of 251 dyad relationships (patient and caregiver couples).

\section{Measures}

We used the EuroQol questionnaire (EQ-5D) to measure QoL prior to discharge of stroke patients and caregivers [24]. The instrument uses a simple generic measure to aggregate QoL into a single index. The EQ-5D consists of five questions about current health status in five dimensions: mobility, self-care, usual activities, pain/discomfort, and anxiety/depression [24, 25]. Responses were no problems (score of 1), moderate problems (2), and extreme problems (3).

Health care professionals used the Barthel Index to assess the level of stroke patients' disability upon admission $[26,27]$. The Satisfaction with Stroke Care (SASC) [20, 21] and the Caregivers' Satisfaction with Stroke Care (C-SASC) [28] questionnaires were used to measure patients' and caregivers' satisfaction with inpatient stroke care, respectively. Patients and caregivers were asked to complete them prior to discharge. We also included length of hospital stay (scored on discharge by the health care professional) and demographic data of patients and their caregivers, including gender, educational level (range, 1-7), living situation, and caregivers' relationship with the stroke patient [partner (=spouse), child, sibling, other].

\section{Statistical analyses}

Means and standard deviations of all study variables were calculated separately for patients and caregivers. Differences between patients' and caregivers' QoL and the norm for the general population in The Netherlands [29] were 
evaluated with $t$ tests. Independent-sample $t$ tests and analysis of variance (ANOVA) were conducted to investigate associations between independent variables and QoL of patients and caregivers. Due to missing data on one or more of the study variables 211 patient-caregiver couples were left for correlation and multilevel regression analyses. Correlations were computed for all variables for patient and caregiver categories, and between patient and caregiver couples.

We used multilevel modeling to identify the indicators related to the patients' and caregivers' QoL scores. The presence of non-independence between patients and caregivers is determined by measuring the association between the scores of the dyad members. Non-independence was measured with the intraclass correlation [19], which indicated that the non-independence of observations is supported statistically and the dyad should be the unit of analysis. We constructed a two-level hierarchical linear model, where the dependence between patients' and caregivers' QoL scores (level 1) was modeled through dyad membership (level 2).

This model is known as the Actor-Partner Interdependence Model (APIM) [18, 19] and can be viewed as the simplest form of the social relations model [30]. The APIM is a model of dyadic relationships that integrates a conceptual view of interdependence with the appropriate statistical techniques for measuring and testing it [19]. The non-independent components of couple data require dataanalytic strategies tailored to the interpersonal processes occurring in the relationship between actor and partner. Dummy variables were used to acknowledge the differential effects and variances for patients and caregivers within dyads. In doing so, the relationship between the independent variables and QoL was investigated separately for patients and caregivers, explicitly taking into account their mutual dependence.

We report our results in the sequence of analysis. To estimate the relative contributions of independent variables, we calculated the reduction in model deviance from the null (model 1). Next, socio-demographic variables for patients and caregivers, the dyad-level variables describing the relationship between patients and caregivers, patients' disease-related characteristics, and satisfaction with care were included as independent variables (model 2).

The analysis was carried out using SPSS software (ver. 18.0; SPSS, Inc., Chicago, IL, USA) with mixed-models repeated measures. We standardized all independent variables for patients and caregivers separately to enable comparison of the associations within and between them. The deviance statistic quantifies the fit of a model compared with the saturated model (i.e., a model that fits perfectly with the empirical data). The difference in deviance of two nested models had a $\chi^{2}$ distribution with degrees of freedom equal to the number of extra parameters in the larger model. We used likelihood ratio tests to compare the relative fit of the two models. Results were considered statistically significant when two-sided $P$ values were $\leq 0.05$. The percentage of explained variance was computed for patients and caregivers. The percentage of explained patient variance was the relative change in total patient variance, computed by summing the patient and dyad variances. Note that these measures are not independent because dyad variance incorporates both patient and caregiver variance.

\section{Results}

Descriptive statistics

Table 1 shows the means and standard deviations of the measures for patients and caregivers separately. Approximately $50 \%$ of patients and the majority of caregivers $(63 \%)$ were women. The mean age of patients was $69 \pm 14.2$ years and caregivers $59 \pm 14.9$ years. The mean educational levels were 2.7 and 3.3 for patients and caregivers, respectively, indicating 9-10 years of formal education. Caregivers were the patient's partner $(52 \%)$, child $(31 \%)$, or sibling (6\%). The patients' and caregivers' mean QoL scores were

Table 1 Descriptive statistics for patients, caregivers, and dyad characteristics

Mean (SD) or $N(\%)$

\begin{tabular}{lc}
\hline Patient $(n=251)$ & $123(49.8 \%)$ \\
Gender; woman & $69.13(14.24)$ \\
Age & $2.74(1.78)$ \\
Educational level & $165(65.7 \%)$ \\
Marital status; married & $11.61(6.84)$ \\
Disability at admission (Barthel) & $11.96(6.88)$ \\
Length of stay (days) & $18.07(3.22)$ \\
Patients' satisfaction with stroke care & \\
(SASC) & \\
Caregiver ( $n=251)$ & $158(62.9 \%)$ \\
Gender; women & $59.14(14.87)$ \\
Age & $3.34(1.81)$ \\
Educational level & $206(82.1 \%)$ \\
Marital status; married & $18.30(2.89)$ \\
Caregivers' satisfaction with stroke care & \\
Dyad characteristics $(n=251)$ & $134(53.3 \%)$ \\
Patient and caregiver living together & \\
Relationship of caregiver to patient & $130(51.9 \%)$ \\
Partner & $78(31.1 \%)$ \\
Daughter/son & $15(6.0 \%)$ \\
Brother/sister & $28(11.1 \%)$ \\
Other & \\
\hline
\end{tabular}


significantly lower than the general Dutch population: $(0.49 \pm 0.38)[t(250)=-15.52, P=0.000]$ and $(0.74 \pm$ 0.34) $[t(206)=-5.73, P=0.000]$ [29], respectively. Satisfaction with stroke care of patients $(18.07 \pm 3.22)$ and caregivers $(18.30 \pm 2.89)$ are comparable to the findings of Boter and colleagues $(18.0 \pm 3.8)$ [20].

\section{Univariate analyses}

Correlations showed that patients' QoL is positively associated with higher satisfaction with stroke care and a higher Barthel score at admission. Patients' QoL was negatively associated with older age and lengthier hospital stay (Table 2). Caregivers' QoL was positively associated with higher satisfaction with care and higher educational level. Their QoL was negatively associated with their own age and lengthier hospital stay of the stroke patient.

\section{Multilevel analyses}

The results of model 1 are shown in Table 3. As expected, patients had a lower mean QoL. Given the two variance components in the lower level of the model (patient and caregiver variance), intraclass correlation was calculated by dividing the dyad variance by the root of the product of the two separate total variances [i.e., $0.06 / \sqrt{ }(0.06+$ $0.09)(0.06+0.06)=0.46]$. The intraclass correlation coefficient was taken to express the "general" correlation coefficient between patient and caregiver QoL, representing the degree of interdependence in the reported QoL.

The results of the full model (model 2) of the multilevel analysis regarding the background characteristics of patients and caregivers revealed that patients' age $(\beta=$ $-0.15)$ and caregivers' educational level $(\beta=0.09)$ were significantly associated with their respective QoL scores. When looking at the patients' disease-related characteristics, the patients' disability at admission (Barthel score) was significantly related to their QoL $(\beta=0.16)$, and to a lesser extent their caregivers' QoL $(\beta=0.08)$. Length of hospital stay was significantly related only to the patients' QoL $(\beta=-0.09)$. Satisfaction with care was significantly related to patients' QoL $(\beta=0.21)$ and showed a weakly significant relationship with caregivers' QoL $(\beta=0.08)$. In comparison with model 1 , the full model (model 2) showed an improvement by a factor of $124.3(d f=23$, $P=0.000$ ), explaining 40 and $16.7 \%$ of patient and caregiver variance, respectively.

\section{Discussion}

The aim of this study was to gain a better understanding of the relationship between the onset of a stroke and the QoL of stroke patients and their caregivers. In particular, we sought to identify the indicators related to patients' and caregivers' QoL and the effect of satisfaction with inpatient stroke care on their QoL. To our knowledge, this is the first study to use the APIM approach to simultaneously investigate patients' and caregivers' satisfaction with stroke care. This study has presented and illustrated the APIM approach as a means of conceptualizing and measuring interdependence in stroke patients and their caregivers, with a special focus on the assessment of bidirectional effects. There is interdependence in a relationship when observations of two individuals-in

Table 2 Correlations between independent variables and quality of life ( $N=211$ dyads)

\begin{tabular}{|c|c|c|c|c|c|c|c|c|c|}
\hline & 1 & 2 & 3 & 4 & 5 & 6 & 7 & 8 & 9 \\
\hline \multicolumn{10}{|l|}{ Patient } \\
\hline \multicolumn{10}{|l|}{ 1. Age } \\
\hline 2. Education & $-0.21 * *$ & & & & & & & & \\
\hline 3. Disability at admission (Barthel) & $-0.31 * *$ & 0.07 & & & & & & & \\
\hline 4. Length of hospital stay & $0.16^{*}$ & -0.04 & $-0.54 * *$ & & & & & & \\
\hline $\begin{array}{l}\text { 5. Patients' satisfaction with stroke care } \\
\text { (SASC) }\end{array}$ & -0.09 & 0.05 & $0.24 * *$ & $-0.17 * *$ & & & & & \\
\hline 6. Quality of life (EQ-5D) & $-0.31 * *$ & 0.08 & $0.61 * *$ & $-0.48 * *$ & $0.35 * *$ & & & & \\
\hline \multicolumn{10}{|l|}{ Caregiver } \\
\hline 7. Age & $0.24 * *$ & -0.11 & -0.10 & -0.03 & -0.09 & -0.03 & & & \\
\hline 8. Education & -0.07 & $0.42 * *$ & 0.00 & 0.05 & 0.02 & -0.01 & $-0.34 * *$ & & \\
\hline $\begin{array}{l}\text { 9. Caregivers' satisfaction with stroke care } \\
\text { (C-SASC) }\end{array}$ & -0.02 & 0.07 & 0.07 & -0.11 & $0.33^{* *}$ & 0.12 & 0.11 & -0.12 & \\
\hline 10. Quality of life (EQ-5D) & -0.05 & $0.14^{*}$ & $0.31 * *$ & $-0.19 * *$ & 0.10 & $0.45 * *$ & $-0.19 * *$ & $0.22 * *$ & 0.08 \\
\hline
\end{tabular}

$* P<0.05 ; * * P<0.01$ (two-tailed) 
Table 3 Multilevel regression analyses $(N=211$ dyads $)$

\begin{tabular}{|c|c|c|c|c|}
\hline \multirow[t]{2}{*}{ Model } & \multicolumn{2}{|l|}{ (Model 1) } & \multicolumn{2}{|l|}{ (Model 2) } \\
\hline & $\beta$ & SE & $\beta$ & SE \\
\hline \multicolumn{5}{|l|}{ Patient } \\
\hline Constant & $0.49 * *$ & 0.02 & $0.53 * *$ & 0.14 \\
\hline Gender $^{\mathrm{a}}$ & & & -0.05 & 0.04 \\
\hline Age & & & $-0.15^{* *}$ & 0.06 \\
\hline Education & & & -0.00 & 0.02 \\
\hline Marital status ${ }^{\mathrm{b}}$ & & & 0.01 & 0.06 \\
\hline Living with caregiver $^{\mathrm{c}}$ & & & -0.07 & 0.07 \\
\hline Caregiver is partner ${ }^{\mathrm{c}}$ & & & 0.00 & 0.10 \\
\hline Disability at admission (Barthel) & & & $0.16^{* *}$ & 0.03 \\
\hline Length of hospital stay & & & $-0.09 * *$ & 0.03 \\
\hline Patients' satisfaction with stroke care (SASC) & & & $0.21 * *$ & 0.06 \\
\hline \multicolumn{5}{|l|}{ Caregiver } \\
\hline Constant & $0.74 * *$ & 0.02 & $0.62 * *$ & 0.15 \\
\hline Gender $^{\mathrm{a}}$ & & & $0.10^{\#}$ & 0.05 \\
\hline Age & & & -0.09 & 0.07 \\
\hline Education & & & $0.09 * *$ & 0.03 \\
\hline Marital status ${ }^{\mathrm{b}}$ & & & 0.00 & 0.08 \\
\hline Living with patient ${ }^{\mathrm{c}}$ & & & 0.07 & 0.09 \\
\hline Patient is partner ${ }^{\mathrm{c}}$ & & & 0.04 & 0.07 \\
\hline Disability at admission (Barthel) & & & $0.08 *$ & 0.04 \\
\hline Length of hospital stay & & & -0.00 & 0.03 \\
\hline Caregivers' satisfaction with stroke care (C-SASC) & & & $0.08^{\#}$ & 0.05 \\
\hline Dyad variance & 0.06 & 0.01 & 0.03 & 0.01 \\
\hline Patient variance & 0.09 & 0.01 & 0.06 & 0.01 \\
\hline Caregiver variance & 0.06 & 0.01 & 0.07 & 0.01 \\
\hline$-2 \log$ likelihood & 333.23 & & 208.93 & \\
\hline Explained patient variance $(\%)$ & & & & 30.8 \\
\hline Explained caregiver variance $(\%)$ & & & & 9.1 \\
\hline Intraclass correlation & 0.46 & & & 0.32 \\
\hline
\end{tabular}

our case stroke patients and their caregivers - are linked or correlated. Such linkages and interdependence of scores calls for use of an APIM approach [19].

Satisfaction with care was related to the QoL of patients and caregivers. Higher satisfaction is associated with higher QoL outcomes for both groups. Furthermore, patients' disability at admission and length of hospital stay were significantly related to patients' QoL. Osberg et al. [4] also found a relationship between disability at admission and patients' QoL. In addition to their study, this study showed that patients' disability at admission was related to caregivers' QoL. Length of stay and caregivers' QoL were not significantly related. In line with previous findings [2], patients' age was significantly related to their QoL. No relationship was found between QoL and educational level of the patient. Older caregivers with lower educational levels were significantly associated with lower QoL scores.
Earlier research on caregiver's age and QoL outcomes showed mixed results: some studies found that caregiver age was not related [31, 32]; some did [33].

Patients' survival of a stroke and caregiving for stroke patients are complex and multidimensional activities. Their nature and determinants evolve over time. We did not investigate whether caregivers had prior caregiving experience, how much time they spend on their caregiving task, their perception of caregiving stress and how this might affect their QoL. We were unable to take into account the impact of changes (e.g., change of mind regarding satisfaction with care or the impact of clinical improvement of patients) over time. Although we did not investigate caregivers' burden and depressive feelings, the EQ-5D instrument we used includes the assessment of anxiety and depressive feelings [24, 25]. Our study was restricted to Dutch stroke services, which limits the applicability of our findings. Our results should be 
confirmed through the use of the APIM approach to investigate QoL of patients and caregivers in other hospital settings and countries.

Patients' and caregivers' mean QoL scores were lower than the Dutch population at large [29]. Delivery of hospital stroke care that supports patients and caregivers by meeting their needs and demands is expected to improve QoL [20, 21], and our study confirmed that satisfaction with care was indeed related to the QoL of both. Higher satisfaction is associated with higher QoL outcomes for both groups. Professionals should treat patients and caregivers with kindness and respect and carefully attend their personal needs to prevent decrease in satisfaction with care of patients and caregivers. Besides providing supportive care that meets the needs and demands of both parties, stroke services should provide sufficient therapy and information about the causes and nature of a stroke [20].

\section{Conclusion}

The APIM distinguished the different roles of patients and caregivers while acknowledging the interdependence of their QoL scores. Satisfaction with care was identified as an important indicator for the QoL of stroke patients and their caregivers, a finding that should be noted by professionals providing stroke services.

Acknowledgments The research was supported by a grant provided by the Netherlands Organisation for Health Research and Development [ZonMw, Grant Number 1010.0015 (95-02-195-15)].

Open Access This article is distributed under the terms of the Creative Commons Attribution Noncommercial License which permits any noncommercial use, distribution, and reproduction in any medium, provided the original author(s) and source are credited.

\section{References}

1. Schweikert, B., Hahmann, H., \& Leidl, R. (2006). Validation of the EuroQoL questionnaire in cardiac rehabilitation. Heart, 92, 62-67.

2. Ahlsio, B., Britton, M., Murray, V., \& Theorell, T. (1984). Disablement and quality of life after stroke. Stroke, 15, 886-890.

3. King, R. B. (1996). Quality of life after stroke. Stroke, 27, 1467-1472.

4. Osberg, J. S., DeJong, G., Haley, S. M., Seward, M. L., McGinnis, G. E., \& Germaine, J. (1988). Predicting long-term outcome among post-rehabilitation stroke patients. American Journal of Physical Medicine and Rehabilitation, 67, 94-103.

5. Viitanen, M., Fugl-Meyer, K. S., Bernspang, B., \& Fugl-Meyer, A. R. (1988). Life satisfaction in long term survivors after stroke. Scandinavian Journal of Rehabilitation Medicine, 20, 17-24.

6. Duncan, P. W., Jorgensen, H. S., \& Wade, D. T. (2000). Outcome measures in acute stroke trials: a systematic review and some recommendations to improve practice. Stroke, 31(1429), 1438.
7. Anderson, R. (1988). The contribution of informal care to the management of stroke. International Journal of Disability Studies, 10, 107-112.

8. Wade, D. T., \& Langton-Hewer, R. (1985). Hospital admission for acute stroke care: who, for how long, and to what effect. Journal of Epidemiology and Community Health, 39, 347-352.

9. Wilkinson, P. R., Wolfe, C. D. A., Warburton, F. G., Rudd, A. G., Howard, R. S., Ross Russell, R. W., et al. (1997). A long-term follow-up of stroke patients. Stroke, 28, 507-512.

10. Addington-Hall, J., Lay, M., Altmann, D., \& McCarthy, M. (1998). Community care for stroke patients in the last year of their life: results of a national retrospective survey of surviving family, friends and officials. Health and Social Care in the Community, 6, 112-119.

11. Scholte, O. P., Reimer, W. J. M., de Haan, R. J., Pijeneborg, J. M. A., Limburg, M., \& van den Bos, G. A. M. (1998). The burden of caregiving in partners of long term stroke survivors. Stroke, 29, 1605-1611.

12. Anderson, C., Linton, J., \& Stewart-Wynne, E. (1995). A population-based assessment of the impact and burden of caregiving for long-term stroke survivors. Stroke, 26, 843-849.

13. Dennis, M., O'Rourke, S., Lewis, S., Sharpe, M., \& Warlow, C. (1998). A quantitative study of the emotional outcome of people caring for stroke survivors. Stroke, 8, 7-17.

14. Greveson, G. C., Gray, C. S., French, J. M., \& James, O. F. (1991). Long term outcomes for patients and caregivers following hospital admission for stroke. Age and Ageing, 20, 337-344.

15. Brugge, C., Alexander, H., \& Hagen, S. (1990). Stroke patients' informal caregivers: patient, caregiver, service factors that affect caregiver strain. Stroke, 30, 1478-1485.

16. Kotila, M., Numminen, H., Waltimo, O., \& Kaste, M. (1998). Depression after stroke: result Of the FINNSTROKE study. Stroke, 29, 368-372.

17. Wade, D. T., Legh-Smith, J., \& Langton, H. R. (1986). Effects of living with and looking after survivors of a stroke. British Medical Journal, 293, 368-372.

18. Campbell, L., \& Kashy, D. A. (2002). Estimating actor, partner, and interaction effects for dyadic data using PROC MIXED and HLM: a user-friendly guide. Personal Relationships, 9, 327-342.

19. Kenny, D. A., \& Cook, W. (1999). Partner effects in relationships research: conceptual issues, analytic difficulties, and illustrations. Personal Relationships, 6, 433-448.

20. Boter, H., de Haan, R. J., \& Rinkel, G. L. E. (2003). Clinimetric evaluation of a satisfaction with Stroke-Care questionnaire. Journal of Neurology, 250, 534-541.

21. Pound, P., Gompertz, P., \& Ebrahim, S. (1994). Patients' satisfaction with stroke services. Clinical Rehabilitation, 8, 7-17.

22. Nieboer, A., Pepels, R., Have, L., van der Kool, T., \& Huijsman, R. (2005). Stroke Services Gespiegeld, Hoofdrapport [Comparison of Stroke Services, main report]. Den Haag, NL: ZonMW.

23. Cramm, J. M., \& Nieboer, A. P. (2011). Professionals' views on factors contributing to multidisciplinary stroke team success. International Journal of Integrated Care, 11, 1-8.

24. EuroQol Group. (1990). EuroQol: a new facility for the measurement of health-related quality of life. Health Policy, 16, 199-208.

25. Brooks, R. (1996). EuroQol: the current state of play. Health Policy, 37, 53-72.

26. Mahoney, F. I., \& Barthel, D. W. (1965). Functional evaluation: the Barthel Index. Maryland State Medical Journal, 14, 61-65.

27. de Haan, R., Limburg, M., Schuling, J., Broeshart, J., Jonkers, L., \& van Zuylen, P. (1993). Klinimetrische evaluatie van de Barthel-index, een maat voor beperkingen in het dagelijks functioneren. Nederlands Tijdschrift voor Geneeskunde, 37, 917-921.

28. Cramm, J. M., Strating, M. M. H., \& Nieboer, A. P. (2011). Validation of the caregivers' Satisfaction with Stroke Care 
Questionnaire: C-SASC hospital scale. Journal of Neurology, 258(6), 1008-1012.

29. Stolk, E. A., Krabbe, P., \& Busschbach, J. (2007). Using the internet to collect EQ-5D norm scores: a valid alternative? In: Proceedings of the 24th Scientific Plenary Meeting of the EuroQol Group. Kijkdui-The Hague, The Netherlands, September 13-15.

30. Snijders, T. A. B., \& Kenny, D. A. (1999). The social relations model for family data: a multilevel approach. Personal Relationships, 6, 471-486.
31. Draper, B. M., Poulos, C. J., Cole, A., Poulos, R. G., \& Ehrlich, F. (1992). A comparison of care-givers for elderly stroke and dementia victims. Journal of American Geriatrics Society, 40, 896-901.

32. Ross, S., \& Morris, R. G. (1988). Psychological adjustment of the spouses of aphasic stroke patients. International Journal of Rehabilitation Research, 11, 383-386.

33. Schulz, R., Tompkins, C. A., \& Rau, M. T. (1988). A longitudinal study of psychosocial impact of stroke on primary support persons. Psychology and Aging, 3, 131-141. 